\title{
Gravitational wave from axionlike particle inflation
}

\author{
Wei Cheng $\odot,{ }^{1,2}$ Tao Qian, ${ }^{3}$ Qing Yu, ${ }^{4}$ Hua Zhou, ${ }^{4}$ and Rui-Yu Zhou ${ }^{1,4, *}$ \\ ${ }^{1}$ School of Science, Chongqing University of Posts and Telecommunications, \\ Chongqing 400065, People's Republic of China \\ ${ }^{2}$ State Key Laboratory of Theoretical Physics, Institute of Theoretical Physics, \\ Chinese Academy of Sciences, Beijing 100190, People's Republic of China \\ ${ }^{3}$ Department of Physics, Nanjing University, \\ 22 Hankou Road, Nanjing 210093, People's Republic of China \\ ${ }^{4}$ Department of Physics, Chongqing University, Chongqing 401331, People's Republic of China
}

(Received 8 April 2021; revised 24 September 2021; accepted 1 October 2021; published 8 November 2021)

\begin{abstract}
In this paper, we investigate gravitational waves (GWs) from the axionlike particle inflation by applying the multinature inflation model. The model parameter $n$ determines the exit way of inflation, the number of phase transitions (PTs), and the number of multiples of inflation. In the inflation exit with PTs, there is a lower bound for parameter $n$ to reach the corresponding multiples of inflation, but the magnitude of $n$ is negligible for the physical observations, such as $n_{s}, r$, and $\Omega_{\mathrm{GW}} h^{2}$. GWs can be generated in two ways for the inflation exit with PTs, one derives from quantum fluctuations, which dominate the low-frequency regions, and these GWs can be detected by the Ultimate-Decigo detector at around $10^{-1} \mathrm{~Hz}$. However, the other one derives from PTs, which dominate the high-frequency regions, and it is expected to be detected by the future higher-sensitivity 3DSR GW detector.
\end{abstract}

DOI: 10.1103/PhysRevD.104.103502

\section{INTRODUCTION}

With the first discovery of gravitational waves (GWs) from a binary neutron star system by the Laser Interferometer Gravitational Wave Observatory (LIGO) [1] and by the Virgo detector [2], the primary GWs from inflation are increasingly the focus of theorists and experimentalists. The inflationary scenario not only explains the problem in the hot big bang universe gracefully, i.e., the flatness puzzles, the horizon puzzles, and the monopole puzzles (refer to Refs. [3-11]), but also predict the near scale invariance which is strongly supported by the anisotropies of cosmic microwave background $(\mathrm{CMB})$ radiation [12].

Typically, in the inflationary models with broken slow-roll conditions [13-20], the primary GWs come only from the quantum fluctuations (QFs) during inflation [21-24], and its intensity is related to the tensor-to-scalar ratio $r$ of inflation $[25,26]$. However, the $r$ predicted by the theory, in this case, is so small that weak GWs will be generated [27], which brings great challenges for experimental detection [28].

\footnotetext{
Corresponding author. zhoury@cqupt.edu.cn

Published by the American Physical Society under the terms of the Creative Commons Attribution 4.0 International license. Further distribution of this work must maintain attribution to the author(s) and the published article's title, journal citation, and DOI. Funded by SCOAP ${ }^{3}$.
}

Alternatively, in the literature [29-31], a new way to end inflation has been proposed, i.e., the inflationary end of the phase transition method. This method also has the natural function of reheating the Universe through the collision of true vacuum bubble walls and converting it to radiation. Here are two scalar fields in this inflationary model; one of the fields is responsible for driving the inflation of the Universe, whereas the other field can indeed percolate the true vacuum from the metastable one and complete the phase transitions (PTs) and end inflation simultaneously. In this case, the source of GWs includes quantum fluctuations during inflation as well as the PT that will produce considerable GWs [32-35]. This makes it possible to detect GWs during inflation.

In this work, a singlet axionlike particle (ALP) is applied to drive cosmic inflation and to terminate the inflation through the PT simultaneously, and we then investigate in detail the GWs produced by the ALP inflation. The multinatural inflation (MNI) [36,37] based on natural inflation (NI) [38] can increase the feasible parameter space of the NI that is shrunk by the latest CMB data [12]. The inflation of MNI has a good property, shift symmetry, which allows an ALP to act as the inflaton [37,39]. Besides, it also keeps the flatness of potential energy, which is essential for driving the expansion of the Universe and generating density perturbations. Moreover, MNI can provide multiple false vacuums due to the multiple cosine functions in the potential of MNI. When the slow-roll inflation proceeds, the inflation first falls 
into the false vacuum, the vacuum bubbles then collide with each other, and the inflation finally transits into the true vacuum. This provides the possibility for MNI of an ALP to complete cosmic inflation and to end inflation through PT simultaneously. We calculate the GWs for the period of ALP inflation that will consist of two parts, namely, the QF GWs and the PT GWs.

The remaining parts of the paper are organized as follows. In Sec. II, we present the multi-NI model and study the inflation exit from the PT. In Sec. III, the GWs from both QF and PT are in detail discussed. Section IV is a summary for this work.

\section{AXIONLIKE PARTICLE INFLATION}

The minimal axionlike particle inflation derived from a multinatural inflation is the simplest and most economical inflation model. In this model, inflation can be terminated in two different ways: One is that the slow-rolling condition breaks directly to stop inflation, which has been widely studied. Another way to stop inflation is through a PT, which can be achieved by changing the related parameter to obtain the false vacua. The minimal axionlike particle inflation potential is defined as follows $[36,37]$ :

$$
V(\phi)=\Lambda^{4}\left[\cos \left(\frac{\phi}{f_{\phi}}+\theta\right)-\frac{\kappa}{n^{2}} \cos \left(n \frac{\phi}{f_{\phi}}\right)\right]+C,
$$

where $\Lambda$ is related to the inflation scale and $f_{\phi}$ represents the decay constant. We set the relative phase $\theta=\pi / 2$ for creating false vacua between the maximum and minimum values of adjacent potential and take the coefficient $\kappa=n^{2}$ for equalizing the energy difference between two adjacent vacua. We require the minimum of the potential at $\phi=\pi / 2$; thus, the rational number $n$ is a multiple of 4 . And $n$ shall determine the tunneling events of the PT. With each PT, the Universe will expand by a certain number of times, so the size of $n$ also determines the number of times the Universe will expand. As the tunneling events of the PT should be more than 200 with a grand unified theory scale inflation [34]; thus, the rational number $n$ should be at least 404. $C=2 \Lambda^{4}$ is a constant that shifts the minimum of the potential to zero.

For the convenience of presentation, we take a small value for $n=20$ and show the ALP inflation potential in Fig. 1, in which the dashed cyan line with arrows represents the direction of inflation and phase transitions. The inflaton first rolls to the first false vacuum point $B$ from the highest point $A$ of potential, then reaches the lower vacuum point through a phase transition, and finally reaches the true vacuum point $C$.

During the slow-roll inflation, the kinetic energy of inflaton is much less than that of the potential energy, and the change rate of kinetic energy should much less than that

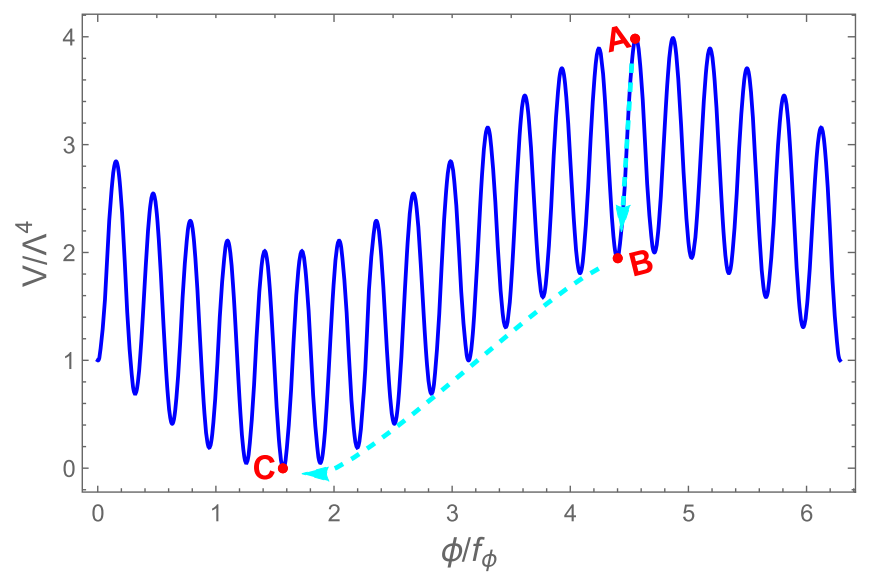

FIG. 1. Schematic diagram of ALP inflation and PT with $n=20$.

of the cosmic expansion, which can be characterized by the so-called slow-roll conditions:

$$
\begin{gathered}
\varepsilon(\phi) \equiv \frac{M_{\mathrm{pl}}^{2}}{2}\left(\frac{V^{\prime}(\phi)}{V(\phi)}\right)^{2} \ll 1, \\
\eta(\phi) \equiv M_{\mathrm{pl}}^{2}\left(\frac{V^{\prime \prime}(\phi)}{V(\phi)}\right) \ll 1,
\end{gathered}
$$

where the prime denotes the derivative with respect to $\phi$.

The slow-roll inflation stops when the slow-roll conditions are broken, i.e., $\varepsilon=1$ or $|\eta|=1$. In the usual method, inflation stops when the slow-roll conditions are broken as the Universe expands. In the ALP inflation exited with PT, the PT takes place before the slow-roll conditions are broken, and the slow-roll condition is broken after the PT. This can also be referred to Refs. [31,40]. More specifically, the slow-roll conditions are still held at the beginning of PT, i.e., $\varepsilon\left(\phi_{B}\right) \ll 1$ and $\eta\left(\phi_{B}\right) \ll 1$ with $\phi_{B}$ bing the field value of the first vacuum, which will bring a lower limit for the decay constant $f_{\phi}$ with a fixed $n$. As an example, we will show two cases as follows:

$$
\begin{aligned}
& f \gg \sqrt{4.39742 \times 10^{31}}, \quad \text { for } n=404 ; \\
& f \gg \sqrt{7.10613 \times 10^{30}}, \quad \text { for } n=2000 .
\end{aligned}
$$

There are two observable quantities, i.e., scalar spectral index $n_{s}=1-6 \varepsilon+2 \eta$ and the tensor-to-scalar ratio $r=16 \varepsilon$, which are tightly constrained by the Planck data combined with $\mathrm{CMB}$ observations as [12]

$$
\begin{gathered}
n_{s}=0.9649 \pm 0.0042, \\
r<0.10(95 \% \text { C.L. }) .
\end{gathered}
$$

For calculating them at the moment of horizon crossing, we need to know the value of the field where inflation starts, $\phi_{*}$, which is usually determined by applying for the $e$-folding number. Numerical calculation shows that the 

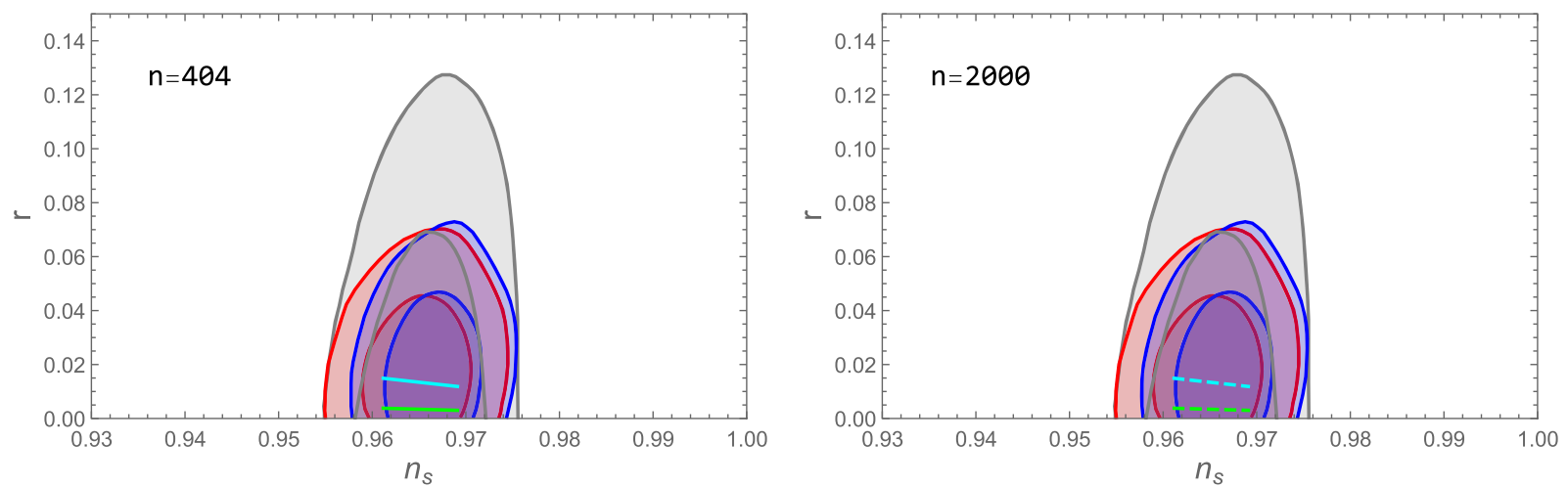

FIG. 2. ALP inflation predictions in $\left(n_{s}, r\right)$ planes with $n=404$ and $n=2000$. Gray, red, and blue shadows stand for [TT, TE, $\mathrm{EE}+$ lowE + lensing], [TT, TE, EE + lowE + lensing + BK15], and [TT, TE, EE + lowE + lensing + BK15 + BAO] constraints, respectively [12].

contributions of the $e$-folding number from the highest potential energy point $\phi_{A}$ to the first vacuum point $\phi_{B}$ are negligible; thus, we are approximating $\phi_{*}$ around the highest potential energy point $\phi_{A}$.

There is another important experimental observation, i.e., amplitude of scalar fluctuations $\Delta_{\mathcal{R}}^{2}$ :

$$
\Delta_{\mathcal{R}}^{2}=\frac{1}{24 \pi^{2} M_{\mathrm{pl}}^{4}} \frac{V\left(\phi_{A}\right)}{\varepsilon\left(\phi_{A}\right)} .
$$

where $\Delta_{\mathcal{R}}^{2} \simeq e^{3.098} \times 10^{-10} \simeq 2.2 \times 10^{-9}$ [41], which will give out the inflation scale $\Lambda$.

Given the above constraints, we fix $n=404,2000$ for the rest of analysis. The scalar spectral index $n_{s}$ and the tensor-to-scalar ratio $r$ are shown in Fig. 2. The green and cyan lines (dashed) represent that $\phi_{*}$ are $602.9 \frac{\pi f_{\phi}}{404}$ and $602.8 \frac{\pi f_{\phi}}{404}\left(2998.9 \frac{\pi f_{\phi}}{2000}\right.$ and $\left.2998.8 \frac{\pi f_{\phi}}{2000}\right)$, respectively. Both $r$ and $n_{s}$ are almost independent of $n$, and the larger $\phi_{*}$, the smaller $r$. To see the specific values more clearly, the upper and lower limits of the corresponding other parameters are listed in Table I.

\section{GRAVITATIONAL WAVES FROM INFLATION}

\section{A. GWs from quantum fluctuations}

During the ALP inflation, the quantum fluctuations of inflaton will lead to the perturbations in energy and momentum, which will transmit to the metric in terms of Einstein's equation, in which the tensor perturbations of the metric will produce the so-called QF GWs. Generally, the initial tensor power spectrum (spectrum at $\tau=0$ ) $P_{h}(k)$ can be parameterized as a power-law form [42], i.e.,

$$
P_{h}(k)=r k^{2} A_{R}\left(k_{0}\right)\left(\frac{k}{k_{0}}\right)^{n_{t}},
$$

where $n_{t}$ is the power index of tensorial spectrum and the value of scalar power spectrum $A_{R}\left(k_{0}\right)=2.371 \times 10^{-9}$ with $k_{0}=0.002 \mathrm{Mpc}^{-1}[41,43]$.

Furthermore, the present spectrum of energy density in terms of $P_{h}(k)$ can be written as [26]

$$
\Omega_{\mathrm{GW}, \mathrm{QF}}(k)=\frac{P_{h}(k)}{12 H^{2}\left(\tau_{0}\right)} \times T^{2}\left(k, \tau_{0}\right),
$$

where the current Hubble parameter $H\left(\tau_{0}\right)$ is taken as $67.8 \mathrm{~km} \mathrm{~s}^{-1} \mathrm{Mpc}^{-1}$, the current conformal time $\tau_{0}=1.41 \times 10^{4} \mathrm{Mpc}$, and the last part $T\left(k, \tau_{0}\right)$ is the transfer function as follows [44-48]:

$$
T\left(k, \tau_{0}\right)=\frac{3 \Omega_{m} j_{1}\left(k \tau_{0}\right)}{k \tau_{0}} \sqrt{1.0+1.36 \frac{k}{k_{e q}}+2.50\left(\frac{k}{k_{e q}}\right)^{2}},
$$

TABLE I. Two sets of benchmark points with $n=404,2000$ for $f_{\phi}(\mathrm{GeV})$ and $10^{-15} \Lambda(\mathrm{GeV})$, in which the scalar spectral index $n_{s}=0.9649 \pm 0.0042$ and $r$ are also presented.

\begin{tabular}{lcccc}
\hline \hline & $n=404$ & $n=404$ & $n=2000$ & $n=2000$ \\
\hline$\phi_{*}$ & $603.9 \frac{\pi f_{\phi}}{404}$ & $603.8 \frac{\pi f_{\phi}}{404}$ & $2998.9 \frac{\pi f_{\phi}}{2000}$ & $2998.8 \frac{\pi f_{\phi}}{2000}$ \\
$f_{\phi}$ & {$\left[10^{21.5388}, 10^{21.5910}\right]$} & {$\left[10^{21.5373}, 10^{21.5895}\right]$} & {$\left[10^{22.2335}, 10^{22.5889]}\right.$} & {$\left[10^{22.2319}, 10^{22.2841]}\right.$} \\
$10^{-15} \Lambda$ & {$[5.62998,5.30161]$} & {$[7.99456,7.52826]$} & {$[5.62942,5.30107]$} & {$[7.99479,7.52848]$} \\
$r$ & {$[0.00385,0.00303]$} & {$[0.01508,0.01186]$} & {$[0.00385,0.00302]$} & {$[0.01509,0.01186]$} \\
\hline \hline
\end{tabular}


where matter density $\Omega_{m}=0.308$, the spherical Bessel function of the first kind $j_{1}\left(k \tau_{0}\right) \sim \frac{1}{\sqrt{2} k \tau_{0}}$, and $k_{e q}=$ $0.073 \Omega_{m} h^{2} \mathrm{Mpc}^{-1}$ is the wave number corresponding to the mode that entered the horizon at the equality of matter and radiation.

Applying the input parameters in Table I and Eq. (9), we show the GWs from the QF during the ALP inflation in Fig. 3, in which the left and right parts stand for the QF GW with $n=404$ and $n=2000$, respectively. The QF GW first decreases with the increase of frequency $f$ and then remains almost constant from frequency $f \sim 10^{-16} \mathrm{~Hz}$. Those two lines with $\phi_{*}=604.9 \pi f / n$ and $\phi_{*}=2998.9 \pi f / n$ $\left(\phi_{*}=604.8 \pi f / n\right.$ and $\left.\phi_{*}=2998.8 \pi f / n\right)$ almost overlap each other due to the same value of tensor-to-scalar ratio $r$ that are shown in Table I, which also implies the QF GWs are not sensitive to the parameter $n$. When we fix the parameter $n$, the QF GWs will increase with the decrease of $\phi_{*}$.

\section{B. GWs from phase transitions}

Cosmological PTs as one of sources of the GWs in the early Universe is currently the focus of most attention. During the PTs, the bubbles begin to nucleate and expand as the Universe cools. And the collision of bubbles and motion of the surrounding cosmic fluid can produce a stochastic $\mathrm{GW}$ background which could be detected by the GW detector. The electroweak phase transition of Standard Model is proved not a true PT but a crossover [49,50]. Many new physical models [51-59] could provide a PT at electroweak scale. Consequently, GWs experiments [60-73] furnish a new way toward new physics in the early Universe.

As we emphasized earlier, in our model, ALP inflation exited through bubble nucleations rather than the direct breach of slow-roll conditions. There is a similar inflation characteristic between the studied ALP inflation ends with PT in this paper and the chain inflation [37,74]; i.e., when the inflation falls into the first false vacuum, the Universe will tunnel rapidly through a series of phase transitions, until it reaches the true vacuum. Each PT occurs in the form of multibubble collisions and is accompanied by the release of energy $\delta \epsilon$, and this energy is equal to the energy difference between the adjacent vacua. For a much more detailed discussion, refer to Refs. [31,74]. As Refs. [75,76] are the study of PT GWs produced by the multibubble collisions with false vacuum and true vacuum energy during single-phase transitions, the GWs for a single PT can be expressed as [75]

$$
\Omega_{m} h^{2}(f)= \begin{cases}\Omega h^{2}\left(f_{m}\right)\left(\frac{f}{f_{m}}\right)^{2.8} & \left(f \leq f_{m}\right), \\ \Omega h^{2}\left(f_{m}\right)\left(\frac{f}{f_{m}}\right)^{-1.8} & \left(f \geq f_{m}\right),\end{cases}
$$

where the peak frequency today $f_{m}=\frac{3 \times 10^{-8}}{\chi} e^{-((n-2) /}$ $2-m-1) \chi\left(\frac{g_{*}}{100}\right)^{1 / 6}\left(\frac{T_{e}}{1 \mathrm{GeV}}\right)$ with the temperature of the Universe at the end of ALP inflation being $T_{e}=\frac{1}{1-e^{-\chi}}\left(\frac{30 \delta \epsilon}{g_{*} \pi^{2}}\right)^{1 / 4}$. The total number of relativistic degrees of freedom is taken to be $g_{*} \simeq 100$. And the amplitude $\Omega h^{2}\left(f_{m}\right)$ at this peak frequency has the following form:

$$
\begin{aligned}
\Omega h^{2}\left(f_{m}\right)= & 10^{-6}\left(1-e^{-\chi}\right)^{4} \chi^{2} e^{-4((n-2) / 2-m-1) \chi} \\
& \times\left(\frac{100}{g_{*}}\right)^{1 / 3} .
\end{aligned}
$$

Thus, the total PT energy density of the GWs is given by a sum of the multiple tunneling events:

$$
\Omega_{\mathrm{GW}, \mathrm{PT}} h^{2}(f)=\sum_{m=1}^{(n-2) / 2-1} \Omega_{m} h^{2}(f) .
$$

We show the PT GWs in Fig. 4, in which the left and right parts stand for the PT GWs with $n=404$ and $n=2000$, respectively. The PT GWs have almost identical peaks and shapes for different $n$, which also implies the PT GWs are insensitive to $n$, but the energy spectrum $\Omega_{\mathrm{GW}, \mathrm{PT}} h^{2}$ sharp is shifted about 5 times to the left as $\phi_{*}$
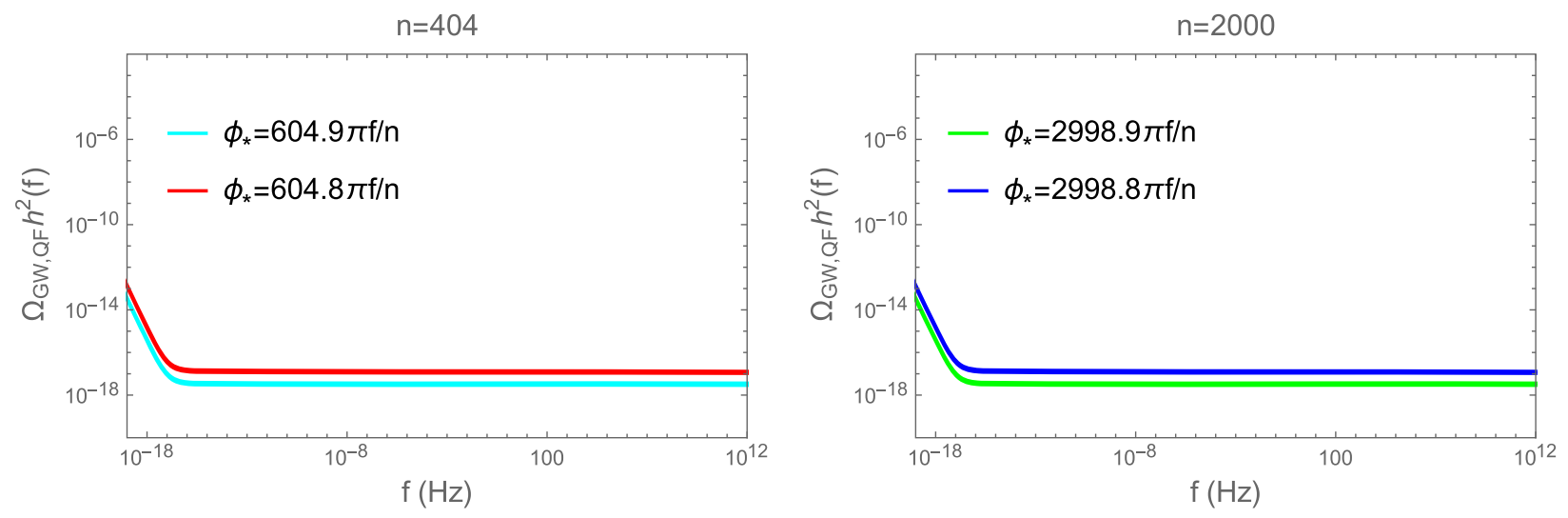

FIG. 3. The spectrum of GW from the quantum fluctuations during the ALP inflation. 

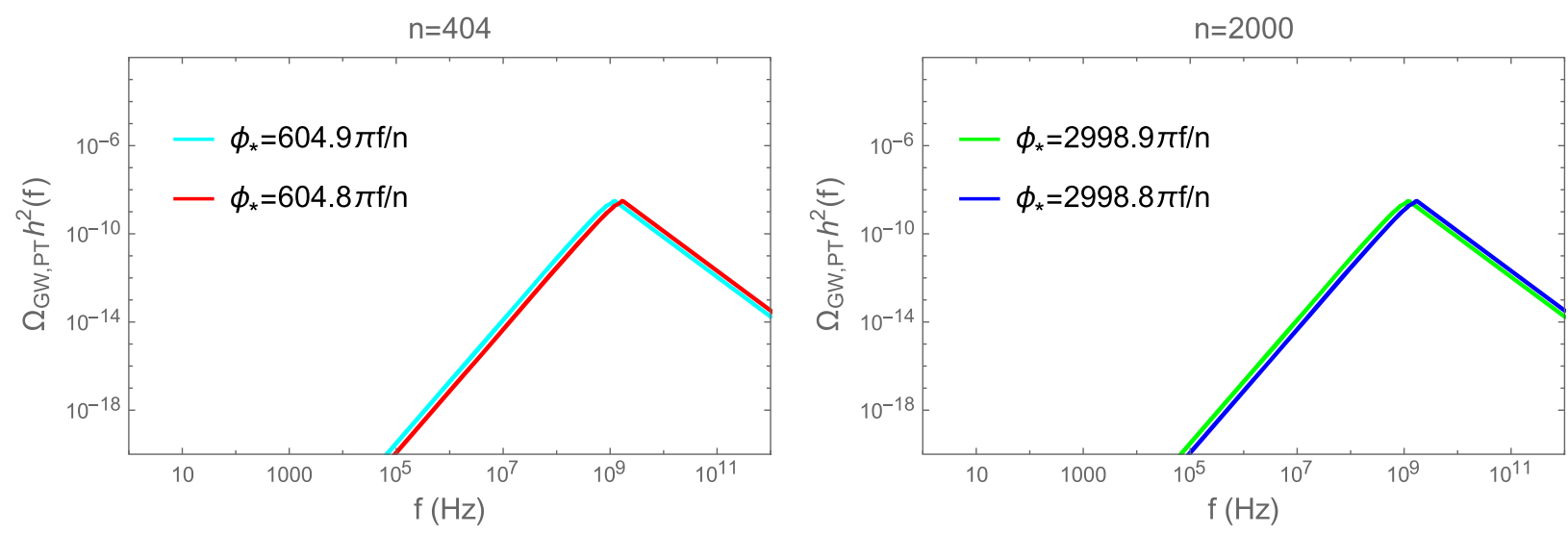

FIG. 4. The spectrum of PT GWs from the ALP inflation.

increases. We can see that the GWs produced by PTs during inflation are the high-frequency GWs. Compared to the previous QF GWs that dominates the low-frequency regions, the PT GWs will dominate the high-frequency regions.

\section{Total GWs}

After acquiring the QF GWs and the PT GWs during the ALP inflation, we can now analyze the total GWs. According to Eqs. (9) and (13), it can be expressed as follows:

$$
\Omega_{\mathrm{GW}, \mathrm{tot}} h^{2}=\Omega_{\mathrm{GW}, \mathrm{PT}} h^{2}+\Omega_{\mathrm{GW}, \mathrm{QF}} h^{2} .
$$

The total GWs are shown in Fig. 5. We find that, at ultralow frequency $f \sim\left(10^{-19}, 10^{-17}\right) \mathrm{Hz}$, all the predictions of the GWs meet the CMB limits [77,78], while near the frequency $f \sim\left(10^{-1}\right) \mathrm{Hz}$, the Ultimate-Decigo detector [79] can search for all of the GW scenarios that we predict.

For the ultrahigh-frequency GWs, there is a peak of GWs at around $10^{10} \mathrm{~Hz}$. To make it easier to compare with experiments, one can study the characteristic amplitude $h_{c}(f)$ that is related with the total GWs $\Omega_{\mathrm{GW}, \text { tot }} h^{2}$, as follows:

$$
h_{c}(f) \approx 1.263 \times 10^{-18}(1 \mathrm{~Hz} / f) \sqrt{\Omega_{\mathrm{GW}, \mathrm{tot}} h^{2}} .
$$

The predicted characteristic amplitudes $h_{c}(f)$ are shown in Fig. 6, in which the red and blue shadows represent the current upper bounds from direct $\mathrm{GW}$ detection experiments and the CMB Rayleigh-Jeans tail constraint, respectively, the magenta line is the building 3DSR GW detection experiments, and the orange dashed line is the nucleosynthesis amplitude upper limit. The results at around $10^{10} \mathrm{~Hz}$ cannot be directly detected by the current experiment but meet the CMB Rayleigh-Jeans tail constraint and nucleosynthesis amplitude limit. As it almost reaches the detection range of the building 3DSR GW detector [80,81], we believe the predicted GWs can be tested by the future higher-sensitivity 3DSR GW detector. Concerning possibilities for detection at
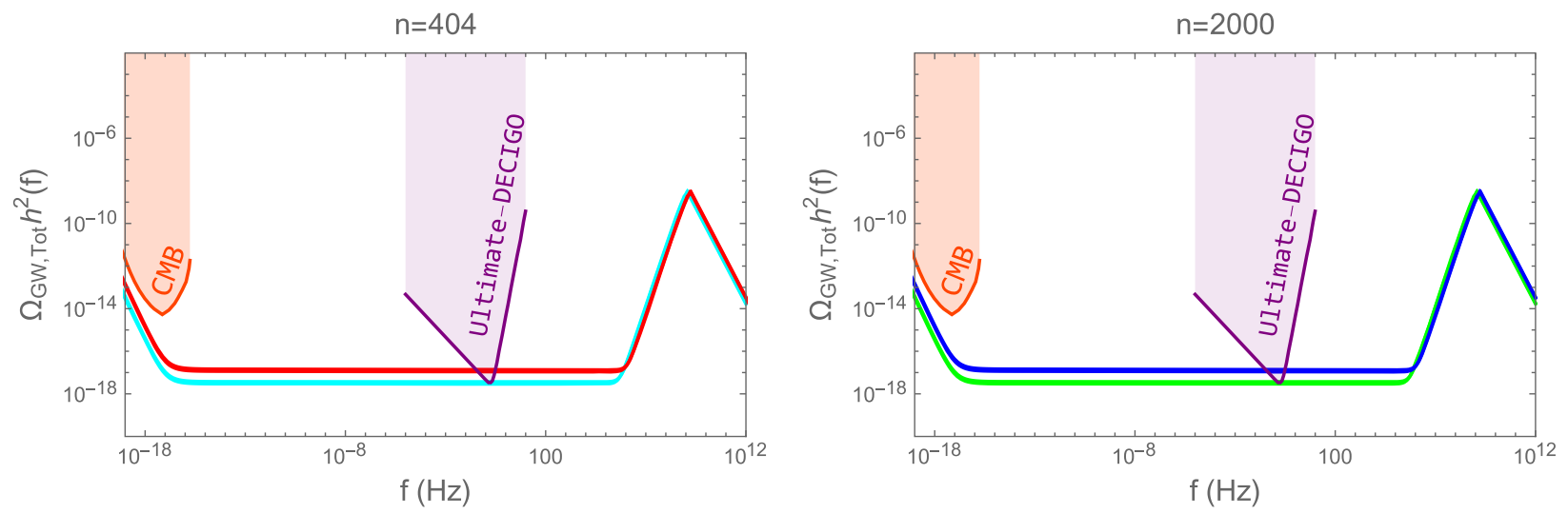

FIG. 5. The spectrum of total GWs from the ALP inflation. The CMB observation makes a limit in the low frequency [77,78], while the Ultimate-Decigo detector [79] can provide a test for the GWs in the high frequency. 

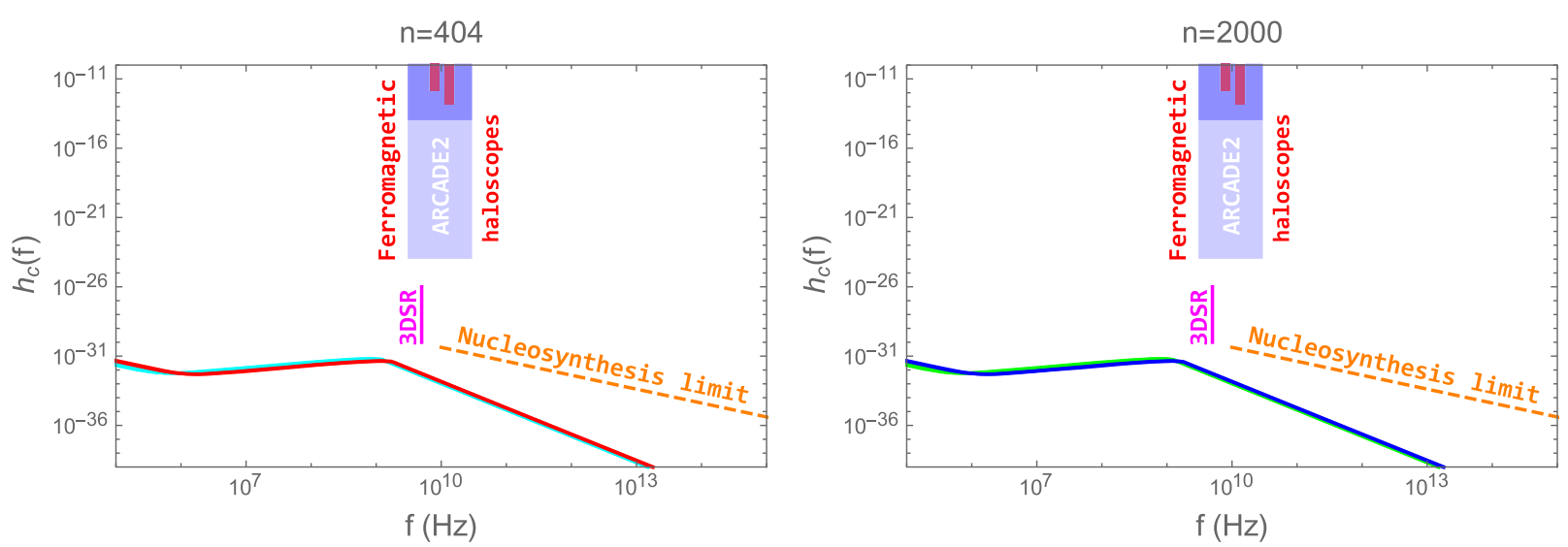

FIG. 6. The total characteristic amplitude $h_{c}(f)$ as a function of $f$. The red and blue shadows represent the current upper bounds from direct GW detection experiments and the CMB Rayleigh-Jeans tail constraint, respectively. The magenta line is the building 3DSR GW detection experiments. The orange dashed line is the nucleosynthesis amplitude upper limit.

high frequency, for more details, refer to the review in Ref. [82].

\section{SUMMARY}

In this paper, we reexamined the minimal axionlike particle inflation derived from a multinatural inflation. We find that the model parameter $n$ may have three main physical meanings: (i) Whether $n$ is equal to 1 or not determines the exit way of inflation. When $n=1$, inflation will end through the slow-roll condition being broken with the expansion of the Universe. While $n>1$, there will be some false vacuum in the potential, which makes it possible for PTs to end inflation. (ii) The size of $n$ determines the number of phase transitions. (iii) With each PT, the Universe will expand by a certain number of times, so the size of $n$ also determines the number of times the Universe will expand.

PTs usually produce sizable GWs that may be detected by the future GW detectors; thus, we studied in detail the GWs from the inflation ending with PTs, i.e., $n>1$. As the PT events need more than 200 , therefore, the model parameter $n$ should not be less than 404 . We investigate two scenarios $(n=404,2000)$ and find that the magnitude of $n$ is negligible for the physical observations, such as $n_{s}, r$, and $\Omega_{\mathrm{GW}} h^{2}$, which can be obtained from the corresponding figures.

In the inflation exit with PTs scenario, the GWs will consist of two parts; i.e., GWs are generated by QF during inflation and by PTs finished the inflation. The generated GWs by QF dominate the low-frequency part, while the GWs generated by PTs dominate the high-frequency part. At $f \sim 10^{-1} \mathrm{~Hz}$, the generated GWs can be detected by Ultimate-Decigo [79], while in the extremely highfrequency part, it is expected to be detected by the future higher-sensitivity 3DSR designed by Chongqing University $[80,81]$.

\section{ACKNOWLEDGMENTS}

This work has been supported by the National Science Foundation of China under Grant No. 12047564, the Fundamental Research Funds for the Central Universities under Grant No. 2020CDJQY-Z003, and the China Postdoctoral Science Foundation under Grants No. 2019TQ0329 and No. 2020M670476.
[1] J. Aasi et al. (LIGO Scientific Collaboration), Advanced LIGO, Classical Quantum Gravity 32, 115012 (2015).

[2] F. Acernese et al. (Virgo Collaboration), Advanced Virgo: A second-generation interferometric gravitational wave detector, Classical Quantum Gravity 32, 024001 (2015).

[3] A. H. Guth, The inflationary Universe: A possible solution to the horizon and flatness problems, Phys. Rev. D 23, 347 (1981).
[4] A. Albrecht and P. J. Steinhardt, Cosmology for Grand Unified Theories with Radiatively Induced Symmetry Breaking, Phys. Rev. Lett. 48, 1220 (1982).

[5] A. D. Linde, A new inflationary universe scenario: A possible solution of the horizon, flatness, homogeneity, isotropy and primordial monopole problems, Phys. Lett. 108B, 389 (1982). 
[6] A. D. Linde, Particle Physics and Inflationary Cosmology, Contemp. Concepts Phys. Vol. 5 (Harwood, Chur, Switzerland, 1990).

[7] A. R. Liddle and D. H. Lyth, The cold dark matter density perturbation, Phys. Rep. 231, 1 (1993).

[8] A. Riotto, Inflation and the theory of cosmological perturbations, ICTP Lect. Notes Ser. 14, 317 (2003), http://users .ictp.it/ pub_off/lectures/lns014/Riotto/Riotto.pdf.

[9] V. Mukhanov, Physical Foundations of Cosmology (Cambridge University Press, Cambridge, England, 2005).

[10] S. Nojiri and S. D. Odintsov, Unified cosmic history in modified gravity: From $\mathrm{F}(\mathrm{R})$ theory to Lorentz non-invariant models, Phys. Rep. 505, 59 (2011).

[11] S. Nojiri, S. D. Odintsov, and V. K. Oikonomou, Modified gravity theories on a nutshell: Inflation, bounce and latetime evolution, Phys. Rep. 692, 1 (2017).

[12] Y. Akrami et al. (Planck Collaboration), Planck 2018 results. X. Constraints on inflation, Astron. Astrophys. 641, A10 (2020).

[13] R. N. Lerner and J. McDonald, Gauge singlet scalar as inflaton and thermal relic dark matter, Phys. Rev. D 80, 123507 (2009).

[14] S. Zheng, Dynamical generation of the weak scale and inflation in high-scale supersymmetry, arXiv:1405.2775.

[15] J. B. Munoz and M. Kamionkowski, Equation-ofstate parameter for reheating, Phys. Rev. D 91, 043521 (2015).

[16] A. Aravind, M. Xiao, and J. H. Yu, Higgs portal to inflation and fermionic dark matter, Phys. Rev. D 93, 123513 (2016); Erratum, Phys. Rev. D 96, 069901 (2017).

[17] W. Cheng and L. Bian, From inflation to cosmological electroweak phase transition with a complex scalar singlet, Phys. Rev. D 98, 023524 (2018).

[18] W. Cheng and L. Bian, Higgs inflation and cosmological electroweak phase transition with $\mathrm{N}$ scalars in the postHiggs era, Phys. Rev. D 99, 035038 (2019).

[19] S. D. Odintsov and V. K. Oikonomou, Aspects of axion $F(R)$ gravity, Europhys. Lett. 129, 40001 (2020).

[20] V. K. Oikonomou, Unifying inflation with early and late dark energy epochs in axion $F(R)$ gravity, Phys. Rev. D 103, 044036 (2021).

[21] S. Matarrese, S. Mollerach, and M. Bruni, Second order perturbations of the Einstein-de Sitter universe, Phys. Rev. D 58, 043504 (1998).

[22] N. Bartolo, S. Matarrese, A. Riotto, and A. Vaihkonen, The maximal amount of gravitational waves in the curvaton scenario, Phys. Rev. D 76, 061302 (2007).

[23] M. Biagetti, M. Fasiello, and A. Riotto, Enhancing inflationary tensor modes through spectator fields, Phys. Rev. D 88, 103518 (2013).

[24] M. Biagetti, E. Dimastrogiovanni, M. Fasiello, and M. Peloso, Gravitational waves and scalar perturbations from spectator fields, J. Cosmol. Astropart. Phys. 04 (2015) 011.

[25] L. A. Boyle and P. J. Steinhardt, Probing the early universe with inflationary gravitational waves, Phys. Rev. D 77, 063504 (2008).

[26] X. J. Liu, W. Zhao, Y. Zhang, and Z. H. Zhu, Detecting relic gravitational waves by pulsar timing arrays: Effects of cosmic phase transitions and relativistic free-streaming gases, Phys. Rev. D 93, 024031 (2016).

[27] A. A. Starobinsky, A new type of isotropic cosmological models without singularity, Phys. Lett. 91B, 99 (1980).

[28] Y. T. Wang, Y. Cai, and Y. S. Piao, Phase-transition sound of inflation at gravitational waves detectors, Phys. Lett. B 789, 191 (2019).

[29] A. D. Linde, Eternal extended inflation and graceful exit from old inflation without Jordan-Brans-Dicke, Phys. Lett. B 249, 18 (1990).

[30] F. C. Adams and K. Freese, Double field inflation, Phys. Rev. D 43, 353 (1991).

[31] A. Ashoorioon, Exit from inflation with a first-order phase transition and a gravitational wave blast, Phys. Lett. B 747, 446 (2015).

[32] C. Caprini, M. Hindmarsh, S. Huber, T. Konstandin, J. Kozaczuk, G. Nardini, J. M. No, A. Petiteau, P. Schwaller, G. Servant et al., Science with the space-based interferometer eLISA. II: Gravitational waves from cosmological phase transitions, J. Cosmol. Astropart. Phys. 04 (2016) 001.

[33] P. Binetruy, A. Bohe, C. Caprini, and J. F. Dufaux, Cosmological backgrounds of gravitational waves and eLISA/ NGO: Phase transitions, cosmic strings and other sources, J. Cosmol. Astropart. Phys. 06 (2012) 027.

[34] A. Ashoorioon and K. Freese, Gravity waves from chain inflation, arXiv:0811.2401.

[35] Y. Di, J. Wang, R. Zhou, L. Bian, R. G. Cai, and J. Liu, Magnetic Field and Gravitational Waves from the FirstOrder Phase Transition, Phys. Rev. Lett. 126, 251102 (2021).

[36] M. Czerny and F. Takahashi, Multi-natural inflation, Phys. Lett. B 733, 241 (2014).

[37] F. Takahashi and W. Yin, ALP inflation and big bang on Earth, J. High Energy Phys. 07 (2019) 095.

[38] F. C. Adams, J. R. Bond, K. Freese, J. A. Frieman, and A. V. Olinto, Natural inflation: Particle physics models, power law spectra for large scale structure, and constraints from COBE, Phys. Rev. D 47, 426 (1993).

[39] R. Daido, F. Takahashi, and W. Yin, The ALP miracle: Unified inflaton and dark matter, J. Cosmol. Astropart. Phys. 05 (2017) 044.

[40] M. Cortes and A. R. Liddle, Viable inflationary models ending with a first-order phase transition, Phys. Rev. D 80, 083524 (2009).

[41] P. Ade et al. (Planck Collaboration), Planck 2015 results. XX. Constraints on inflation, Astron. Astrophys. 594, A20 (2016).

[42] H. V. Peiris et al. (WMAP Collaboration), First year Wilkinson Microwave Anisotropy Probe (WMAP) observations: Implications for inflation, Astrophys. J. Suppl. 148, 213 (2003).

[43] P. A. R. Ade et al. (Planck Collaboration), Planck 2015 results. XIII. Cosmological parameters, Astron. Astrophys. 594, A13 (2016).

[44] M. S. Turner, M. J. White, and J. E. Lidsey, Tensor perturbations in inflationary models as a probe of cosmology, Phys. Rev. D 48, 4613 (1993). 
[45] Y. Zhang, Y. Yuan, W. Zhao, and Y. T. Chen, Relic gravitational waves in the accelerating Universe, Classical Quantum Gravity 22, 1383 (2005).

[46] S. Chongchitnan and G. Efstathiou, Prospects for direct detection of primordial gravitational waves, Phys. Rev. D 73, 083511 (2006).

[47] M. Giovannini, Stochastic backgrounds of relic gravitons: A theoretical appraisal, PMC Phys. A 4, 1 (2010).

[48] S. Kuroyanagi, C. Gordon, J. Silk, and N. Sugiyama, Forecast constraints on inflation from combined CMB and gravitational wave direct detection experiments, Phys. Rev. D 81, 083524 (2010); Erratum, Phys. Rev. D 82, 069901 (2010).

[49] K. Kajantie, M. Laine, K. Rummukainen, and M. E. Shaposhnikov, Is There a Hot Electroweak Phase Transition at $\mathrm{m}(\mathrm{H})$ Larger or Equal to $\mathrm{m}(\mathrm{W})$ ? Phys. Rev. Lett. 77, 2887 (1996).

[50] F. Csikor, Z. Fodor, and J. Heitger, Endpoint of the Hot Electroweak Phase Transition, Phys. Rev. Lett. 82, 21 (1999).

[51] A. V. Kotwal, M. J. Ramsey-Musolf, J. M. No, and P. Winslow, Singlet-catalyzed electroweak phase transitions in the $100 \mathrm{TeV}$ frontier, Phys. Rev. D 94, 035022 (2016).

[52] P. Huang, A. J. Long, and L. T. Wang, Probing the electroweak phase transition with Higgs factories and gravitational waves, Phys. Rev. D 94, 075008 (2016).

[53] F. P. Huang, Z. Qian, and M. Zhang, Exploring dynamical $\mathrm{CP}$ violation induced baryogenesis by gravitational waves and colliders, Phys. Rev. D 98, 015014 (2018).

[54] C. W. Chiang, Y. T. Li, and E. Senaha, Revisiting electroweak phase transition in the standard model with a real singlet scalar, Phys. Lett. B 789, 154 (2019).

[55] T. Li and Y. F. Zhou, Strongly first order phase transition in the singlet fermionic dark matter model after LUX, J. High Energy Phys. 07 (2014) 006.

[56] N. Chen, T. Li, Y. Wu, and L. Bian, Complementarity of the future $e^{+} e^{-}$colliders and gravitational waves in the probe of complex singlet extension to the standard model, Phys. Rev. D 101, 075047 (2020).

[57] J. M. Cline, G. Laporte, H. Yamashita, and S. Kraml, Electroweak phase transition and LHC signatures in the singlet majoron model, J. High Energy Phys. 07 (2009) 040.

[58] W. Chao, First order electroweak phase transition triggered by the Higgs portal vector dark matter, Phys. Rev. D 92, 015025 (2015).

[59] L. Bian, H. K. Guo, and J. Shu, Gravitational waves, baryon asymmetry of the universe and electric dipole moment in the CP-violating NMSSM, Chin. Phys. C 42, 093106 (2018); Erratum, Chin. Phys. C 43, 129101 (2019).

[60] C. Caprini, M. Chala, G. C. Dorsch, M. Hindmarsh, S. J. Huber, T. Konstandin, J. Kozaczuk, G. Nardini, J. M. No, K. Rummukainen et al., Detecting gravitational waves from cosmological phase transitions with LISA: An update, J. Cosmol. Astropart. Phys. 03 (2020) 024.

[61] B. Abbott et al. (LIGO Scientific and Virgo Collaborations), Observation of Gravitational Waves from a Binary Black Hole Merger, Phys. Rev. Lett. 116, 061102 (2016).

[62] J. Aasi et al. (LIGO Scientific and Virgo Collaborations), Characterization of the LIGO detectors during their sixth science run, Classical Quantum Gravity 32, 115012 (2015).

[63] E. Thrane and J. D. Romano, Sensitivity curves for searches for gravitational-wave backgrounds, Phys. Rev. D 88, 124032 (2013).

[64] B. Abbott et al. (LIGO Scientific and Virgo Collaborations), Search for the isotropic stochastic background using data from Advanced LIGO second observing run, Phys. Rev. D 100, 061101 (2019).

[65] G. Janssen, G. Hobbs, M. McLaughlin, C. Bassa, A. Deller, M. Kramer, K. Lee, C. Mingarelli, P. Rosado, S. Sanidas, A. Sesana, L. Shao, I. Stairs, B. Stappers, and J. Verbiest, Gravitational wave astronomy with the SKA, Proc. Sci., AASKA14 (2015) 037 [arXiv:1501.00127].

[66] G. Desvignes et al., High-precision timing of 42 millisecond pulsars with the European Pulsar Timing Array, Mon. Not. R. Astron. Soc. 458, 3341 (2016).

[67] J. P. W. Verbiest et al., The international pulsar timing array: First data release, Mon. Not. R. Astron. Soc. 458, 1267 (2016).

[68] J. Luo et al. (TianQin Collaboration), TianQin: A spaceborne gravitational wave detector, Classical Quantum Gravity 33, 035010 (2016).

[69] X. Gong et al., Descope of the ALIA mission, J. Phys. Conf. Ser. 610, 012011 (2015).

[70] P. Amaro-Seoane et al. (LISA Collaboration), Laser interferometer space antenna, arXiv:1702.00786.

[71] S. Hild et al., Sensitivity studies for third-generation gravitational wave observatories, Classical Quantum Gravity 28, 094013 (2011).

[72] M. Punturo et al., The Einstein Telescope: A third-generation gravitational wave observatory, Classical Quantum Gravity 27, 194002 (2010).

[73] B. P. Abbott et al. (LIGO Scientific Collaboration), Exploring the Sensitivity of Next Generation Gravitational Wave Detectors, Classical Quantum Gravity 34, 044001 (2017).

[74] K. Freese and D. Spolyar, Chain inflation: 'Bubble bubble toil and trouble', J. Cosmol. Astropart. Phys. 07 (2005) 007.

[75] A. Kosowsky and M. S. Turner, Gravitational radiation from colliding vacuum bubbles: Envelope approximation to many bubble collisions, Phys. Rev. D 47, 4372 (1993).

[76] S. J. Huber and T. Konstandin, Production of gravitational waves in the nMSSM, J. Cosmol. Astropart. Phys. 05 (2008) 017.

[77] P. D. Lasky, C. M. F. Mingarelli, T. L. Smith, J. T. Giblin, D. J. Reardon, R. Caldwell, M. Bailes, N. D. R. Bhat, S. Burke-Spolaor, W. Coles et al., Gravitational-Wave Cosmology Across 29 Decades in Frequency, Phys. Rev. X 6, 011035 (2016).

[78] A. Sepehri, A. Pradhan, and S. Shoorvazi, $F(R)$ bouncing cosmology with future singularity in brane-anti-brane system, Astrophys. Space Sci. 361, 58 (2016).

[79] K. Yagi and N. Seto, Detector configuration of DECIGO/ BBO and identification of cosmological neutron-star binaries, Phys. Rev. D 83, 044011 (2011).

[80] F. Y. Li, M. X. Tang, and D. P. Shi, Electromagnetic response of a Gaussian beam to high frequency relic gravi- 
tational waves in quintessential inflationary models, Phys. Rev. D 67, 104008 (2003).

[81] M. 1. Tong, Y. Zhang, and F. Y. Li, Using polarized maser to detect high-frequency relic gravitational waves, Phys. Rev. D 78, 024041 (2008).
[82] N. Aggarwal, O. D. Aguiar, A. Bauswein, G. Cella, S. Clesse, A. M. Cruise, V. Domcke, D. G. Figueroa, A. Geraci, M. Goryachev et al., Challenges and opportunities of gravitational wave searches at $\mathrm{MHz}$ to $\mathrm{GHz}$ frequencies, arXiv:2011.12414. 